\title{
BMJ Open Protocol for a qualitative synthesis of barriers and facilitators in implementing guidelines for diagnosis of tuberculosis
}

\author{
Eleanor Ochodo, ${ }^{1}$ Tamara Kredo, $^{2}$ Taryn Young, ${ }^{1,2}$ Charles Shey Wiysonge ${ }^{1,2}$
}

To cite: Ochodo E, Kredo T, Young T, et al. Protocol for a qualitative synthesis of barriers and facilitators in implementing guidelines for diagnosis of tuberculosis. BMJ Open 2017;7:e013717. doi:10.1136/ bmjopen-2016-013717

- Prepublication history and additional material are available. To view these files please visit the journal online (http://dx.doi. org/10.1136/bmjopen-2016013717).

Received 1 August 2016 Revised 23 March 2017 Accepted 23 March 2017

\section{CrossMark}

${ }^{1}$ Centre for Evidence-based Health Care, Faculty of Medicine and Health Sciences, Stellenbosch University, Cape Town, South Africa

${ }^{2}$ Cochrane South Africa, South African Medical Research Council, Cape Town, South Africa

Correspondence to Dr Eleanor Ochodo; eleanor.ochodo@gmail.com

\section{ABSTRACT}

Introduction Despite the introduction of new tests and guidelines for diagnosis of tuberculosis (TB), worldwide case detection rate of TB is still suboptimal. This could be in part explained by the poor implementation of TB diagnostic guidelines. We aim to identify, appraise and synthesise qualitative evidence exploring the barriers and facilitators to implementing TB diagnostic guidelines.

Methods and analysis A systematic review of qualitative studies will be conducted. Relevant electronic databases will be searched and studies included based on predefined inclusion criteria. We will also search reference lists, grey literature, conduct forward citation searches and contact relevant content experts. An adaptation of the Critical Appraisal Skills Programme tool will be used to assess the methodological quality of included studies. Two authors will review the search output, extract data and assess methodological quality independently, resolving any disagreements by consensus. We will use the thematic framework analysis approach based on the Supporting the Use of Research Evidence thematic framework to analyse and synthesise our data. We will apply the Confidence in the Evidence from Reviews of Qualitative research approach to transparently assess our confidence in the findings of the systematic review.

Ethics and dissemination This protocol has been registered with the International Prospective Register of Systematic Reviews (PROSPER0), registration number CRD42016039790

Trial registration number PROSPERO 2016: CRD42016039790. Available from http://www.crd.york.ac. UK/PROSPERO/

\section{BACKGROUND}

Despite the introduction of new tests and guidelines for diagnosis of tuberculosis (TB), case detection of infected people or people with disease still remains far from optimal. ${ }^{12}$ In 2014, the worldwide case detection rate of TB disease was only $63 \% .{ }^{1}$ It is also approximated that 3 million cases are missed by health systems every year. The need to accurately identify persons with $\mathrm{TB}$ in a population is important in administering correct and timely treatment, in limiting the spread of TB especially the

\section{Strengths and limitations of the study}

- We have prepared this protocol in line with PRISMA_P (Preferred Reporting Items for Systematic Reviews and Meta-analysis Protocols) guidelines.

- We will use rigorous methods to systematically identify, appraise and synthesise the qualitative evidence.

- To minimise selection and reviewer bias, two review authors will perform study identification and data extraction independently.

- This review will be limited by what is reported in the included studies. We will not seek original data.

drug-resistant strains and in avoiding unnecessary mortality. In 2013, a total of 480000 people developed multidrug-resistant TB with 210000 associated deaths. ${ }^{3}$

One reason for the poor case detection of TB could be poor implementation of guidelines to diagnose TB. Such guidelines aim to assist health workers to identify infected or diseased patients by recommending tests and testing algorithms to be used depending on a patient's symptoms and healthcare resources and setting. However, quantitative studies have shown that health workers adhere poorly to TB algorithms, policies or guidelines. ${ }^{4-7}$ For example, a secondary analysis of trial data on health worker adherence to a TB diagnostic algorithm in South Africa, found that the algorithm was only adhered to in $24 \%$ of HIV-infected patients with an initial negative TB test. ${ }^{6}$ Poor health worker adherence or implementation of TB diagnostic guidelines has also been reported in other low-income, ${ }^{78}$ middle-income ${ }^{4910}$ and high-income settings. ${ }^{11}$

To explain the possible reasons for poor implementation or adherence to health guidelines, primary qualitative studies done in various settings have cited possible reasons including health stakeholders (healthcare workers, managers, patients), health system or 
contextual barriers. ${ }^{11-16}$ Systematically collating the findings of primary qualitative studies exploring barriers and facilitators could advance the evidence base and guide implementation approaches. To our knowledge, there is no qualitative systematic review exploring factors affecting the implementation of TB diagnostic guidelines. Such a review could help explain the perspectives from various settings or help generate more generalisable theories or hypotheses. ${ }^{17}$ A Cochrane review looking at the effectiveness of interventions to increase $\mathrm{TB}$ case detection is currently under way. ${ }^{18}$ Our qualitative review may provide greater insights to why those interventions succeed or fail. This information may help guide the implementation of TB diagnostic guidelines and also the design of effective interventions to improve uptake of these guidelines.

\section{OBJECTIVE}

Through this review, we aim to identify, appraise and synthesise qualitative evidence to describe the barriers and facilitators to implementing guidelines on TB diagnosis.

\section{Criteria for considering studies for this review}

We shall include studies that fulfil the following criteria:

\section{Types of studies}

In this systematic review of qualitative studies we will include studies that employ qualitative study designs, for example, case studies, grounded theory, phenomenological studies, mixed methods and ethnographic designs. Any study that uses qualitative methods for data collection such as interviews (individual and focus group), observation and qualitative methods for data analysis such as thematic analysis will be included. We will exclude studies that collect data using qualitative methods but analyse the data quantitatively. We shall exclude studies with included comments from quantitative surveys, editorials and opinion pieces.

\section{Types of participants}

We will include studies that report on the perspectives of health workers, health managers, policymakers, patients, activists, academics and other stakeholders that we will come across in the studies towards implementation of guidelines for TB diagnosis. We define health workers as 'all people engaged in actions whose primary intent is to enhance health' as recommended by the WHO. ${ }^{19}$

\section{Type of setting}

We will include studies from any geographical setting globally and from any setting where TB diagnosis is conducted including healthcare facilities, the community and during home visits.

\section{Types of interventions}

To enhance the applicability of our review, we will include studies that focus on the implementation of a guideline about any diagnostic test, algorithm or strategy for any form of TB including latent TB infection, pulmonary or extrapulmonary disease, drug susceptible or drug resistance disease. We will include any qualitative study that explores the guideline implementation of any diagnostic test, algorithm or strategy whether it is the main focus of the study or nested within the study. We will focus on the implementation of guidelines that form part of the specific context's standard of care. We will apply a broad definition of the term guideline described as 'systematically developed statements to assist practitioner and patient decisions about healthcare for specific clinical circumstances. ${ }^{, 20}$ Studies that explore the implementation of a diagnostic test (eg, Xpert MTB/RIF) or algorithm (eg, smear negative algorithms) or process regardless of whether the term guideline is explicitly mentioned will also be included. Examples of guideline implementation strategies or interventions include those targeted at healthcare organisations (organisational culture, continuous quality improvement), healthcare workers (education, training, audit and feedback, reminders, patient-mediated interventions) and patients (reminders, financial incentives). ${ }^{21} 22$

\section{Types of outcome measures}

The phenomena of interest in this review are attitudes, perspectives and experiences of health stakeholders (eg, health workers, managers, policymakers, patients) when implementing guidelines on TB diagnosis.

\section{Search methods for identification of studies}

We will develop a search strategy using guidelines recommended by the Cochrane Qualitative Research Methods group $^{23}$ and search multiple electronic sources and include studies without date or language restrictions.

The search strategy will incorporate the key terms: 'guidelines', 'tuberculosis', 'implementation', 'attitudes' and their associated synonyms. We will search electronic databases including: MEDLINE, EMBASE, TRIP, The Cochrane Library, CINAHL (Cumulative Index to Nursing and Allied Health Literature) and several regional databases (African Index Medicus, Index Medicus for the Eastern Mediterranean Region, INDMED, HERDIN, Thai Index Medicus, LILACS). The detailed MEDLINE search strategy available in box 1 will be tailored to different databases.

We will also search other resources such as the reference list of included studies, grey literature including government or non-governmental organisation reports, and websites that contain evidence on TB diagnosis including the WHO, Evidence-based Tuberculosis Diagnosis and the International Union Against TB and Lung Disease. We will also conduct forward citation searching of relevant articles using related articles features on PubMed and Google scholar. To access unpublished literature, we will contact relevant experts in the field of TB and implementation of guidelines.

To minimise selection bias, two authors will independently screen the search outputs for potentially eligible studies, compare their selections, and resolve disagreements by discussion and consensus. These authors 


\section{Box 1 Search strategy (for MEDLINE)}

1. 'Tuberculosis/diagnosis' OR 'Tuberculosis/epidemiology' OR 'Tuberculosis/prevention and control'[Mesh]

2. (('Tuberculosis, Multidrug-Resistant/diagnosis'[Mesh] OR 'Tuberculosis, Multidrug-Resistant/epidemiology'[Mesh] OR 'Tuberculosis, Multidrug-Resistant/prevention and control "[Mesh])))

3. ((tuberculosis OR TB OR MDR-TB OR XDR-TB) AND (diagnosis OR diagnostic* OR detection OR RDT* OR screen*) [Title/Abstract])

4. (tuberculosis OR TB OR MDR-TB OR XDR-TB) AND ('active case finding' or ACF) [Title/Abstract]

5. (tuberculosis OR TB OR MDR-TB OR XDR-TB) AND 'point of care' [Title/Abstract]

6. 'Latent Tuberculosis' [Mesh] AND (diagnosis OR diagnostic* OR detection $\mathrm{OR} \mathrm{RDT}^{\star}$ OR screen* or 'active case finding' or ACF or 'point of care' [Title/Abstract])

7. 1 or 2 or 3 or 4 or 5 or 6

8. 'Qualitative Research'[MeSH] OR 'Interviews as Topic'[MeSH]

9. 'Attitude of Health Personnel' [Mesh]

10. Qualitative or survey or 'focus group*' or interview* or questionnaire or experience* or 'mixed method "[Title/Abstract]

11. 'Health services'[MeSH] OR 'Delivery of Health Care'[Mesh] OR 'Health Services Accessibility'[Mesh] OR 'Patient Acceptance of Health Care'[Mesh] OR 'Health Status Disparities'[Mesh] or 'Health Knowledge, Attitudes, Practice'[Mesh]

12. 'Health Personnel/education'[Mesh] OR 'Health Personnel/ manpower'[Mesh] OR 'Health Personnel/organization and administration'[Mesh] OR 'Health Personnel/psychology'[Mesh] OR 'Health Personnel/statistics and numerical data'[Mesh] OR 'Health Personnel/utilization'[Mesh] or Health Personnel/standards [Mesh]

13. 'Healthcare utilization' $O R$ 'healthcare seeking' [Title/Abstract]

14. 'Patients' [Mesh] or 'patient compliance' [MeSH] or 'Consumer Participation'[Mesh] or (stakeholder* or provider* or 'health manager*' or "community-based or academic* or advocacy [Title/ Abstract]

15. barrier* or delay* or facilitat* or enable* or limit* or drawback* or challeng* or stigma* or failure* or constrain ${ }^{\star}$ or factor* or challenge* or attitude* or perspective* [Title/Abstract]

16. 'Delayed Diagnosis'[Mesh] or 'Delayed diagnosis' [Title/Abstract] or 'Diagnostic delay"' [Title/Abstract]

17. 8 or 9 or 10 or 11 or 12 or 13 or 14 or 15 or 16

18. guideline* or 'best practice*' or evidence or EBM) AND (adher* or apply* or application or disseminat* or implement* or introduce* or uptake) [Title/Abstract])

19. Consensus OR Statement OR Algorithm [Title/Abstract]

20. 'Guideline Adherence'[Mesh]

21. 18 or 19 or 20

22. 7 and 17 and 21

23. 22 NOT (case reports[pt] OR editorial[pt] OR comment[pt] OR practice guideline[pt])

will also independently screen the full text of potentially eligible articles to check if the articles fulfil the inclusion criteria defined by the types of studies, participants, intervention, setting and outcomes. The search results will be presented in the form of a flow diagram as recommended by the Preferred Reporting Items for Systematic Reviews and Meta-analysis (PRISMA). ${ }^{24}$

\section{Data extraction and analysis}

Drawing from the Supporting the Use of Research Evidence (SURE) framework, ${ }^{25}$ we shall develop a structured and standardised data extraction form for extracting data from the selected studies. The SURE framework focuses on barriers to implementing health systems interventions and includes elements on knowledge and skills, health system challenges, and social and political constraints (online supplementary appendix 1). To ensure the integrity of the assessment, we will pilot the data extraction form on at least three studies identified from the list of potentially eligible studies. We will extract data about the first author, publication year, journal, language, participant group (cadre of health worker), setting (country, rural/urban, type of health facility), intervention (type, description and recommendation of guideline, the test, test strategy or algorithm and form of TB focused by the guideline), research methods (method of data collection and analysis, framework used) and outcomes (reported barriers and facilitators and related themes). Two authors will independently extract the data. Any disagreements will be resolved through discussion or by a third reviewer.

\section{Assessment of quality of included studies}

There is currently no consensus on standard criteria to be applied to assess the methodological quality of qualitative studies. For our review, we will use an adaptation of the Critical Appraisal Skills Programme (CASP) quality assessment tool for qualitative studies. ${ }^{17}$ This adapted tool was developed based on the authors' experience in synthesising qualitative studies on adherence to TB treatment. In this light, it may be applicable to our focus on TB diagnostic guidelines. Other systematic reviews of qualitative studies have also used this tool. ${ }^{26} 27$ Two review authors will independently apply the CASP tool. Any disagreements will be resolved though discussion or by a third author. Our adapted CASP checklist will have the following eight questions which will be scored as either Yes, No or Unclear:

1. Is the study setting or context described sufficiently?

2. Is the sampling method clearly described?

3 . Is the data collection method clearly described?

4. Is the method for data analysis clearly described and appropriate?

5. Are the findings or conclusions made supported by adequate evidence?

6. Is there evidence of reflexivity?

7. Does this study demonstrate sensitivity to ethical concerns?

8. Any other concerns?

Studies will not be excluded on the basis of quality. However, we will apply the CASP tool as part of the Confidence in the Evidence from Reviews of Qualitative research (CERQual) approach for assessing confidence in the findings of the systematic review (see below). 


\section{DATA SYNTHESIS}

To analyse and synthesise qualitative data, we will use the thematic framework analysis approach. ${ }^{23}{ }^{27}$ Thematic synthesis is useful where the evidence is likely to be largely descriptive and will enhance our understanding of why the health stakeholders think, feel and behave as they do. We will follow the five stages of framework synthesis to synthesise our qualitative data:

Familiarisation with the data: The first author will begin with familiarisation of the data against the aims of the review and note recurrent themes across the studies.

Identifying a thematic framework: Instead of developing our own a priori framework, we will use the SURE thematic framework to guide our thematic analysis. However, we will adapt this framework based on the emerging themes from our analysis. This framework provides a detailed list of possible factors that could influence the implementation of an intervention.

Indexing: Two review authors will independently read the extracted information to search for themes according to the SURE framework and additional emergent themes. The framework will be revised as new themes emerge. This will be done upon discussion and agreement by the entire author team. All studies will be read until there are no new emerging themes. Coding of the data will be done based on the themes identified in the data. Each primary study will be indexed using the codes related to the themes of the framework. Where appropriate, parts of the studies may be indexed with one or more codes.

Charting: We will sort the data by theme and present the themes in the form of an analysis table (chart). The columns and rows of the table will reflect the studies and related themes and enable us to compare findings of the studies across different themes and subthemes.

Mapping and interpretation: We will use the charts to define the identified concepts and map the range and nature of the phenomena. We will explore associations between the themes to help explain the findings better. We will map and interpret our findings in line with the review objectives and emerging themes.

We will present our findings as a 'Summary of qualitative findings' table that will summarise our key findings, our confidence judgement for each finding and a related explanation of the assessment.

\section{Subgroup analysis and investigation of heterogeneity}

We will assess heterogeneity of findings or differences in views of the included participants by conducting subgroup analyses according to: type of stakeholder (eg, health worker, patient, manager, policymaker) and setting (low-income and middle-income countries vs high-income countries). We will also consider other axes that could emerge as important when synthesising the evidence such as urban/rural or high income/low income setting across both low/middle and high-income countries or cadre of health worker (clinicians vs nurses vs community workers).

\section{Assessment of confidence in the review findings}

We will apply the CERQual approach to transparently assess our confidence in the findings of the systematic review. ${ }^{28}$ This approach draws on the principles of the Grading of Recommendations, Assessment, Development and Evaluation approach. The CERQual approach assesses confidence in the review findings based on four components which include: the methodological limitations of included studies, the relevance of the included studies to the review question, the coherence of the review findings and the adequacy of data contributing to the review findings.

Concerns with any of the four components will be noted for each review finding and taken into consideration when making an overall CERQual assessment of confidence in that finding as either high, moderate, low or very low. The CERQual assessment and written justification will appear in a summary of qualitative findings table.

\section{Reporting of protocol and systematic review}

We have prepared this protocol according to the reporting guideline: Preferred Reporting Items for Systematic Reviews and Meta-analysis Protocols (PRISMA-P) ${ }^{29}$ We will also use the PRISMA ${ }^{24}$ to report the findings of our review. Because PRISMA focuses on reporting of reviews of interventions we will use the relevant items to report findings of our qualitative review.

\section{Ethics and dissemination}

This protocol has been registered with the International Prospective Register of Systematic Reviews (PROSPERO), registration number CRD42016039790. This review is a retrospective study, drawing on data that are publicly available and does not need to undergo a formal ethical review for approval. We will disseminate the findings of this review through publication in a peer-reviewed journal and via conference presentations. We shall also prepare a review summary using the SURE dissemination guides ${ }^{30}$ and disseminate it to groups involved in the translation of TB guidelines into practice in South Africa. We will discuss the findings and applicability of our review in line with our review question, relevance to the implementation of guidelines for TB diagnosis and implications for future research.

Contributors Concept of the study: EO. Drafting of initial protocol: EO. Intellectual input on versions of the protocol: EO, TK, TY, CSW. Approving final draft of protocol: EO, TK, TY, CSW.

Funding This work was supported by the Special Programme for Research and Training in Tropical Diseases (WHO/TDR), grant number B40410 awarded to Dr Eleanor Ochodo. TDR has no role in the design, conduct and interpretation of this review. Dr Eleanor Ochodo's salary is supported through a grant from the Wellcome Trust Foundation. The Wellcome Trust also has no role in the design, conduct and interpretation of this review.

Competing interests None declared.

Provenance and peer review Not commissioned; externally peer reviewed.

Open Access This is an Open Access article distributed in accordance with the Creative Commons Attribution Non Commercial (CC BY-NC 4.0) license, which 
permits others to distribute, remix, adapt, build upon this work non-commercially, and license their derivative works on different terms, provided the original work is properly cited and the use is non-commercial. See: http://creativecommons.org/ licenses/by-nc/4.0/

(C) Article author(s) (or their employer(s) unless otherwise stated in the text of the article) 2017. All rights reserved. No commercial use is permitted unless otherwise expressly granted.

\section{REFERENCES}

1. WHO. Global tuberculosis Report 2015. 2015. http://www.who.int/tb/ publications/global_report/gtbr2015_executive_summary.pdf

2. Alsdurf $\mathrm{H}, \mathrm{Hill} P C$, Matteelli A, et al. The cascade of care in diagnosis and treatment of latent tuberculosis infection: a systematic review and meta-analysis. Lancet Infect Dis 2016;16:1269-78.

3. WHO. The WHO EndTB strategy. 2015. http://www.who.int/tb/ post2015_strategy/en/

4. Shewade HD, Govindarajan S, Sharath BN, et al. MDR-TB screening in a setting with molecular diagnostic techniques: who got tested, who didn't and why? Public Health Action 2015;5:132-9.

5. Shirzadi MR, Majdzadeh R, Pourmalek F, et al. Adherence of the private sector to national tuberculosis guidelines in the islamic Republic of Iran, 2001-02. Eastern Mediterranean health journal = La revue de sante de la Mediterranee orientale = al-Majallah al-sihhiyah li-sharq al-mutawassit 2003;9:796-804.

6. McCarthy KM, Grant AD, Chihota V, et al. Implementation and operational research: what happens after a negative test for tuberculosis? evaluating adherence to TB diagnostic algorithms in South African primary health clinics. J Acquir Immune Defic Syndr 2016;71:e119-26.

7. Zaeh S, Kempker R, Stenehjem E, et al. Improving tuberculosis screening and isoniazid preventive therapy in an HIV clinic in Addis Ababa, Ethiopia. Int J Tuberc Lung Dis 2013;17:1396-401.

8. Alamo ST, Kunutsor S, Walley J, et al. Performance of the new WHO diagnostic algorithm for smear-negative pulmonary tuberculosis in HIV prevalent settings: a multisite study in Uganda. Trop Med Int Health 2012;17:884-95.

9. Satyanarayana S, Subbaraman R, Shete P, et al. Quality of tuberculosis care in India: a systematic review. Int J Tuberc Lung Dis 2015;19:751-63.

10. Oshi DC, Chukwu JN, Nwafor CC, et al. Diagnosis of smear-negative tuberculosis in Nigeria: do health care workers adhere to the national guidelines? Int J Mycobacteriol 2014;3:163-7.

11. Mulder $\mathrm{C}$, Harting $\mathrm{J}$, Jansen $\mathrm{N}$, et al. Adherence by dutch public health nurses to the national guidelines for tuberculosis contact investigation. PLOS One 2012;7:e49649.

12. Cattamanchi A, Miller CR, Tapley A, et al. Health worker perspectives on barriers to delivery of routine tuberculosis diagnostic evaluation services in Uganda: a qualitative study to guide clinic-based interventions. BMC Health Serv Res 2015;15:10.

13. Joseph HA, Shrestha-Kuwahara R, Lowry D, et al. Factors influencing health care workers' adherence to work site tuberculosis screening and treatment policies. Am J Infect Control 2004;32:456-61.

14. Wynne A, Richter S, Banura L, et al. Challenges in tuberculosis care in Western Uganda: health care worker and patient perspectives. Int $J$ Africa Nurs Sci 2014;1:6-10.

15. Gebreegziabher SB, Yimer SA, Bjune GA. Qualitative assessment of challenges in tuberculosis control in West Gojjam Zone, Northwest Ethiopia: health Workers' and tuberculosis control program coordinators' perspectives. Tuberc Res Treat 2016;2016:1-8.

16. Naidoo P, van Niekerk M, du Toit E, et al. Pathways to multidrugresistant tuberculosis diagnosis and treatment initiation: a qualitative comparison of patients' experiences in the era of rapid molecular diagnostic tests. BMC Health Serv Res 2015;15:488.

17. Atkins $\mathrm{S}$, Lewin $\mathrm{S}, \mathrm{Smith} \mathrm{H}$, et al. Conducting a meta-ethnography of qualitative literature: lessons learnt. BMC Med Res Methodol 2008;8:21.

18. Mhimbira FA, Cuevas LE, Dacombe R, et al. Interventions to increase tuberculosis case detection at primary healthcare or community level services. 2015

19. WHO. World Health Report. 2006, 2006.

20. Medicine lo. Institute of Medicine. Clinical practice guidelines: directions for a new program. In: Field MJ, Lohr KN, eds. Washington, DC: The National Academies Press, 1990:168.

21. Grimshaw JM, Thomas RE, MacLennan G, Fraser C, et al. Effectiveness and efficiency of guideline dissemination and implementation strategies. Health Technol Assess 2004;8:84

22. Mazza D, Bairstow P, Buchan H, Chakraborty SP, et al. Refining a taxonomy for guideline implementation: results of an exercise in abstract classification. Implement Sci 2013;8:32.

23. Noyes JPJ, Pearson A, Hannes K, et al; Cochrane handbook: chapter 20: qualitative research and Cochrane reviews, 2016.

24. Moher D, Liberati A, Tetzlaff J, Altman DG, et al. Preferred reporting items for systematic reviews and meta-analyses: the PRISMA statement. PLoS Med 2009;6:e1000097.

25. Collaboration. TSStUoRE. SURE Guides for Preparing and using Evidence-based policy briefs: 5 . identifying and addressing barriers to implementing the Policy Options. version 2.1, updated November 2011: The SURE Collaboration, 2011. http://global.evipnet.org/ SURE-Guides/

26. Ames HMR GC, Lewin S. Parents' and informal caregivers' views and experiences or routine early childhood vaccination communication: qualitative evidence synthesis (Protocol). Cochr Datab Syst Rev 2015;7:CD011787.

27. Glenton C, Colvin CJ, Carlsen B, et al. Barriers and facilitators to the implementation of lay health worker programmes to improve access to maternal and child health: qualitative evidence synthesis. Cochrane Database Syst Rev 2013;10:CD010414.

28. Lewin $\mathrm{S}$, Glenton $\mathrm{C}$, Munthe-Kaas $\mathrm{H}$, et al. Using qualitative evidence in decision making for health and social interventions: an approach to assess confidence in findings from qualitative evidence syntheses (GRADE-CERQual). PLoS Med 2015;12:e1001895.

29. Moher D, Shamseer L, Clarke M, Ghersi D, et al. Preferred reporting items for systematic review and meta-analysis protocols (PRISMA-P) 2015 statement. Syst Rev 2015;4:1.

30. Collaboration TS. SURE guides for preparing and using evidencebased policy briefs. 2011 http://global.evipnet.org/SURE-Guides/ 